\title{
An Ant-based Selection Hyper-heuristic for Dynamic Environments
}

\author{
Berna Kiraz ${ }^{1}$, A. Şima Etaner-Uyar² ${ }^{2}$ and Ender Özcan ${ }^{3}$ \\ 1 Institute of Science and Technology, Istanbul Technical University, Turkey \\ Berna.Kiraz@marmara.edu.tr \\ 2 Department of Computer Engineering, Istanbul Technical University, Turkey \\ etaner@itu.edu.tr \\ 3 School of Computer Science, University of Nottingham, UK \\ Ender.Ozcan@nottingham.ac.uk
}

\begin{abstract}
Dynamic environment problems require adaptive solution methodologies which can deal with the changes in the environment during the solution process for a given problem. A selection hyper-heuristic manages a set of low level heuristics (operators) and decides which one to apply at each iterative step. Recent studies show that selection hyperheuristic methodologies are indeed suitable for solving dynamic environment problems with their ability of tracking the change dynamics in a given environment. The choice function based selection hyper-heuristic is reported to be the best hyper-heuristic on a set of benchmark problems. In this study, we investigate the performance of a new learning hyper-heuristic and its variants which are inspired from the ant colony optimisation algorithm components. The proposed hyper-heuristic maintains a matrix of pheromone intensities (utility values) between all pairs of low level heuristics. A heuristic is selected based on the utility values between the previously invoked heuristic and each heuristic from the set of low level heuristics. The ant-based hyper-heuristic performs better than the choice function and even its improved version across a variety of dynamic environments produced by the Moving Peaks Benchmark generator.
\end{abstract}

\section{Introduction}

Many real world constraint optimisation problems contain a set of components which might change in time separately or concurrently. Some of these components include the problem instance, the objectives and the constraints. A good solution method needs to be adaptive and intelligent to be able to deal with the complexities introduced by such a dynamic environment and track the changes. Branke [2] categorized these changes based on (i) frequency, (ii) severity, (iii) predictability of a change, and (iv) cycle length/cycle accuracy which is a property defining somewhat periodicity and precision of changes. There is a variety of approaches dealing with different types of changes in literature. Most of the 
approaches handling dynamic environment problems are modified from the existing approaches designed for solving static problems based on different strategies. More details on dynamic environments can be found in $[2,5,11,16]$.

In this study, we use an emerging search methodology, namely selection hyper-heuristics for solving dynamic environment problems. A selection hyperheuristic controls a set of low level heuristics, choosing the most appropriate one to apply to a solution in hand and deciding to accept or reject the newly created solution at each step [3]. There are learning hyper-heuristic methodologies as well as the ones which do not utilise any learning at all. An online learning selection hyper-heuristic can incorporate learning either into the heuristic selection, or move acceptance methods. These components attempt to improve performance during the search process. If learning takes place during heuristic selection, frequently, a mechanism is used to score the performance of each low level heuristic. Then a heuristic is chosen based on these scores. Nareyek [12] tested Reinforcement Learning (RL) heuristic selection on Orc Quest and modified logistics domain. Initially, scores are initialised to the same value for all heuristics, e.g., 0. After a chosen heuristic is applied to a solution, if there is improvement, then its score is increased; otherwise it is decreased, e.g. by one. A heuristic is chosen using different mechanisms and the best strategy appears to be selecting the heuristic with maximum score. Cowling et al. [4] investigated the performance of different heuristic selection methods on a real world scheduling problem. One of them is a learning method referred to as Choice Function (CF) which maintains a score for each low level heuristic taking a weighted average of three values; how well it performs individually and as a successor of the previously invoked heuristic and the elapsed time since its last call. Then the heuristic with the maximum score is selected and applied to the current solution at a given step. Drake and Özcan [7] proposed a modified version of CF improving its performance (ICF) in which weights dynamically change, enforcing the search process to go into diversification faster than usual, when the successive moves are non-improving.

There is strong empirical evidence that selection hyper-heuristics work for not only discrete combinatorial problems [3] but also discrete and continuous dynamic environment problems $[9,10,15,14]$, being able to respond to the changes in such an environment rapidly. In this study, we describe a new learning hyperheuristic for dynamic environments, which is designed based on the ant colony optimisation algorithm components. The proposed hyper-heuristic maintains a matrix of pheromone intensities (utility values) between all pairs of low level heuristics. A heuristic is selected based on the utility values between the previously invoked heuristic and each heuristic from the set of low level heuristics. We investigate the performance of the proposed hyper-heuristic controlling a set of parameterised mutation operators for solving the dynamic environment problems produced by the Moving Peaks Benchmark (MPB) generator. 


\section{Proposed Method}

In this study, we propose a selection hyper-heuristic incorporating a novel heuristic selection method, called the Ant based Selection $(A b S)$, which is based on a simple ant colony optimization (ACO) algorithm [6]. Most of the mechanisms used in ACO are adapted within $A b S$. A distinct feature of $A b S$ is that unlike $\mathrm{ACO}, A b S$ is based on a single point based search framework. In most of the selection hyper-heuristics, there is a heuristic selection step followed by an acceptance step. After the heuristic selection step using $A b S$, we employ the generic Improving-and-Equal acceptance scheme, which accepts a new solution of better or equal quality as compared to the previous solution.

Similar to the Choice Function and Reinforcement Learning heuristic selection schemes, $A b S$ also incorporates an online learning mechanism using a matrix of utility values. In $A b S$, each heuristic pair is associated with a pheromone trail value $\left(\tau_{h_{i}, h_{j}}\right)$ which shows the desirability of selecting the $j^{t h}$ heuristic after the application of the $i^{t h}$ heuristic. All pheromone trails are initialized with a small value $\tau_{0} . A b S$ selects a random low-level heuristic at the first step. In the following steps, the most appropriate low-level heuristic is selected and applied to a solution in hand based on the pheromone trail information.

$A b S$ consists of heuristic selection and pheromone update stages. For the first stage, we consider two variants of heuristic selection schemes. In both variants, the heuristic $h_{s}$ with the highest pheromone trail $\left(h_{s}=\max _{i=1 . . k} \tau_{h_{c}, h_{j}}\right)$ is selected with a probability of $q_{0}$ where $h_{c}$ is the previously selected heuristic. Otherwise, methods inspired by two of the mate selection techniques most commonly used in Evolutionary Algorithms [8] are employed to determine the next heuristic to select. In the first variant, like in ACO, the next heuristic is determined based on probabilities proportional to the pheromone levels of each heuristic pair. This is similar to the roulette wheel mate selection in Evolutionary Algorithms. In this method, AbSrw selects the next heuristic $h_{s}$ with a probability which is proportional to the pheromone trail value of $\tau_{h_{c}, h_{s}}$. However, in the second variant (AbSts), the choice of the next heuristic is based on tournament selection. AbSts chooses the next heuristic $h_{s}$ with the highest pheromone trail $\left(h_{s}=\max _{i=1 . . k} \tau_{h_{c}, h_{j}}\right)$.

After selecting a heuristic, pheromone trails are modified. Firstly, pheromone values on the pheromone matrix are decreased by a constant factor (evaporation) for all pairs of heuristics as given in Equation 1.

$$
\tau_{h_{i}, h_{j}}=(1-\rho) \tau_{h_{i}, h_{j}}
$$

where $0<\rho \leq 1$ is the pheromone evaporation rate.

After evaporation, the pheromone value between only $h_{c}$ and $h_{s}\left(\tau_{h_{c}, h_{s}}\right)$ is increased using Equation 2.

$$
\tau_{h_{c}, h_{s}}=\tau_{h_{c}, h_{s}}+\Delta \tau
$$

where $h_{c}$ is the previously selected heuristic and $h_{s}$ is the last selected heuristic. $\Delta \tau$ is the amount of pheromone added and is defined as in Equation 3.

$$
\Delta \tau=1 / f_{c}
$$


where $f_{c}$ is the fitness value of the new solution generated by applying the selected heuristic $h_{s}$.

\section{Experimental Design}

In this study, we perform experiments with our new hyper-heuristic for dynamic environments, combining the Ant-based selection scheme and the Improvingand-Equal acceptance technique. For comparison, we also experiment with previously used selection mechanisms which incorporate some form of online learning and are shown to be successful in dynamic environments [10], namely the Choice Function and Reinforcement Learning. In this paper, we also include an improved version of the Choice Function proposed in [7]. These selection mechanisms are also used together with the Improving-and-Equal acceptance technique.

In the experiments, we used the Moving Peaks Benchmark (MPB) generator [1] to generate the various dynamic environments. In MPB, the height, width and location of the peaks forming the multimodal and multidimensional landscape are changed every $\Delta e$ iterations by adding a normally distributed random variable to the heights and the widths of the peaks and by shifting their locations with a vector of fixed length in a random direction. In some applications, a time-invariant basis function is also included in the generated landscapes. The height_severity, the width_severity and vlength parameters determine the severity of the changes in the heights, the widths and the locations of the peaks respectively. For the fixed parameters of the MPB in all the experiments, we used the settings taken from $[1,2]$ as follows: cone peak function, 5 peaks, 5 dimensions, range of peak heights $\in[30,70]$, range of peak widths $\in[0.8,7.0]$ and range of values in each dimension $\in[0.0,100.0]$. We did not use a basis function and allowed the peak shifts to be uncorrelated. For the $\Delta e$, height_severity, the width_severity and vlength settings we used the ones given in [10] and labeled as EXPSET2. Based on these settings, $\Delta e$ is taken as 6006 fitness evaluations for low frequency (LF), 1001 for medium frequency (MF) and 126 for high frequency (HF); the height_severity, the width_severity and vlength parameters are taken as given in Table 1 which correspond to low severity (LS), medium severity (MS) and high severity (HS) changes.

Table 1. MPB parameter settings for high, medium and low severity changes

\begin{tabular}{c|c|c|c}
\hline \hline Setting & LS & MS & HS \\
\hline vlength & 1.0 & 5.0 & 10.0 \\
height_severity & 1.0 & 5.0 & 10.0 \\
width_severity & 0.1 & 0.5 & 1.0 \\
\hline \hline
\end{tabular}

A real-valued vector corresponds to the coordinates of a point in the search space generated by the MPB. The fitness of a candidate solution at a given time $t$ is given by its error, which is calculated as its distance to the optimum in 
terms of the objective function value at time $t$. Therefore, the goal turns into minimising the error values for a given problem.

The search algorithm moves through the landscape by perturbing a candidate solution at each step to obtain a new one using a parameterized Gaussian mutation, $N\left(0, \sigma^{2}\right)$, where $\sigma$ denotes the standard deviation. We used the same settings for the mutation operators as in [10], which are implemented as seven different standard deviations; $\{0.5,2,7,15,20,25,30\}$. These mutation operators are used as the low-level heuristics in the hyper-heuristic framework.

The parameters of the proposed Ant-based selection scheme are chosen as follows: $\rho$ is set to 0.1. Each entry in the pheromone matrix is initialized to $\tau_{0}=$ $1 / f_{s}$ where $f_{s}$ is the fitness value of initial solution. For $A b S r w$, we experiment with seven $q_{0}$ values: $\{0.0,0.1,0.3,0.5,0.7,0.9,1.0\}$. For AbSts, we consider five tournament size values: $\{2,3,4,5,6\}$ as well as the above given seven $q_{0}$ values. We also included variants of the approaches in which $\Delta \tau$ is updated with a value changing at a slower rate during the pheromone evaporation process. In this case, $\Delta \tau$ is calculated as $\Delta \tau=0.1 *\left(1 / f_{c}\right) . A b S r w$ and AbSts with the slower decrease rates are denoted as $s A b S r w$ and $s A b S t s$, respectively.

The parameter settings of the other heuristic selection methods are done using the same settings proposed in previous studies from the literature. In Reinforcement Learning, the scores of all heuristics are initialized to 15 with lower and upper bounds as 0 and 30 respectively as given in [13]. At each step, the score of a heuristic that improves performance is increased by 1 and otherwise it is decreased by 1 . In Choice Function, $\alpha, \beta$, and $\delta$ are initialized to 0.5 with updates of \pm 0.01 at each iteration as given in [7]. In the Improved Choice Function, $\phi$, which refers $\alpha$ and $\beta$, and $\delta$ are initialized to 0.5. If the heuristic improves performance, the values of $\phi$ are set to 0.99 . Otherwise, the values of $\phi_{t}$ at time $t$ are calculated as $\phi_{t}=\max \left\{\phi_{t-1}-0.01,0.01\right\}$. In addition, $\delta$ is calculated as $\delta_{t}=1-\phi_{t}$

We assume that all programs are made aware when a change in the environment occurs. For the Reinforcement Learning, Choice Function and the Improved Choice Function heuristic selection methods, when a change occurs, the current solution is re-evaluated. For the proposed Ant-based selection scheme, this is not required. The parameters of none of the heuristic selection methods are reset when the environment changes. Due to the nature of the acceptance mechanism, Improving-and-Equal, the first solution candidate generated after each environment change is accepted regardless of its solution quality.

For evaluating the performance of the approaches, we used the offline error metric [2]. The error of a candidate solution at a given time is calculated as its distance to the optimum in terms of the objective function value at that time. The offline error is calculated as a cumulative average of the errors of the best candidate solutions found so far since the last change. At the end of a run, a lower overall offline error value is desired indicating a good performance.

Each run is repeated 100 times for each setting where 20 changes occur, i.e. there are 21 consecutive stationary periods. This means that there are maxIterations $=($ NoOfChanges +1$) *$ ChangePeriod number of iterations 
per run. For analysis of statistical significance of the differences obtained between the results of various approaches, we performed ANOVA tests together with Tukey's HSD at a confidence level of $95 \%$.

\section{Results and Discussion}

In this section we provide the results of the experiments and their discussions. Table 2 summarizes the results of the parameter tuning tests for $q_{0}$ in AbSrw and sAbSrw. In the table, $q_{0}=0.0$ means that the next heuristic is chosen using only the roulette-wheel selection. However, $q_{0}=1.0$ means that roulette wheel selection is not used and always the heuristic with the best score (pheromone value) is chosen to be applied. The results show that there are no statistically significant differences between most cases, however, the best values are provided by different $q_{0}$ values for different frequency-severity pairs. Therefore, to avoid overtuning, we decided to choose a setting which provided an acceptable performance in most of the cases for both approaches. For the rest of the experiments we continued with a setting of $q_{0}=0.5$ for both AbSrw and sAbSrw.

Table 2. Final offline error results of various $q_{0}$ settings for AbSrw and sAbSrw under the tested change frequency-severity pairs

\begin{tabular}{l|c|ccc|ccc|ccc}
\hline \hline \multirow{2}{*}{ Algorithm } & \multirow{2}{*}{$q_{0}$} & \multicolumn{4}{|c|}{ LF } & \multicolumn{3}{c|}{ MF } & \multicolumn{3}{c}{ HF } \\
\cline { 3 - 11 } & & LS & MS & HS & LS & MS & HS & LS & MS & HS \\
\hline \multirow{4}{*}{ AbSrw } & 0.0 & 3.577 & 7.664 & 9.875 & 4.647 & 8.860 & 12.321 & 9.886 & 17.031 & 24.041 \\
& 0.1 & 3.463 & 7.641 & 10.276 & 4.376 & 9.340 & 11.754 & 10.138 & 17.138 & 25.243 \\
& 0.3 & 3.929 & 8.026 & 10.244 & 4.608 & 8.774 & 12.354 & 9.286 & 16.453 & 24.612 \\
& 0.5 & 3.720 & 8.114 & 10.580 & 4.640 & 9.601 & 13.051 & 9.404 & 15.461 & 24.260 \\
& 0.7 & 4.186 & 8.434 & 10.562 & 4.975 & 10.838 & 13.178 & 10.434 & 17.419 & 25.493 \\
& 0.9 & 3.931 & 10.420 & 10.849 & 4.775 & 10.459 & 13.648 & 12.504 & 19.807 & 28.935 \\
& 1.0 & 3.956 & 10.368 & 12.332 & 5.533 & 10.849 & 13.997 & 15.898 & 23.064 & 31.920 \\
\hline \multirow{5}{*}{ AbSrw } & 0.0 & 3.734 & 7.454 & 9.730 & 4.388 & 8.531 & 11.935 & 10.350 & 17.105 & 25.586 \\
& 0.1 & 3.723 & 7.090 & 9.882 & 4.490 & 8.510 & 12.330 & 9.948 & 17.183 & 24.368 \\
& 0.3 & 3.978 & 7.598 & 10.189 & 4.394 & 8.245 & 12.467 & 8.875 & 15.444 & 24.027 \\
& 0.5 & 3.644 & 7.402 & 10.990 & 4.180 & 8.698 & 12.421 & 7.932 & 14.935 & 22.904 \\
& 0.7 & 3.879 & 8.578 & 10.945 & 4.483 & 9.490 & 12.531 & 8.914 & 16.004 & 24.131 \\
& 0.9 & 4.278 & 8.827 & 11.936 & 4.399 & 10.095 & 13.334 & 8.691 & 15.432 & 23.713 \\
& 1.0 & 4.878 & 9.959 & 12.563 & 6.528 & 12.620 & 14.802 & 13.535 & 19.350 & 25.191 \\
\hline \hline
\end{tabular}

Then we performed parameter tuning experiments to set $q_{0}$ and tournament size values for the AbSts and sAbSts variants. Our experiments revealed that for this hyper-heuristic variant, the best settings highly depend on the dynamics of the environment, i.e. the change frequency and severity values. To be able to choose a value which would give a good performance across different types of 
chnages, we calculated the average and standard deviation values for the final offline error results for each change frequency-severity setting over all $q_{0}$ and tournament size combinations, i.e. over 35 combinations each for AbSts and sAbSts. Table 3 lists these results.

Table 3. Average and standard deviation of final offline error values over all $q_{0}$ and tournament size combinations under all tests frequency-severity pairs

\begin{tabular}{c|c|c|c}
\hline \hline \multicolumn{2}{l|}{} & AbSts & sAbSts \\
\hline \multirow{3}{*}{ LF } & LS & $4.061 \mp 0.238$ & $4.121 \mp 0.525$ \\
& MS & $8.911 \mp 0.510$ & $8.997 \mp 0.644$ \\
& HS & $11.291 \mp 0.505$ & $11.577 \mp 0.795$ \\
\hline & LS & $5.361 \mp 0.343$ & $5.106 \mp 0.736$ \\
MF & MS & $10.514 \mp 0.649$ & $10.530 \mp 0.867$ \\
& HS & $13.345 \mp 0.536$ & $13.318 \mp 0.717$ \\
\hline \multirow{3}{*}{ HF } & LS & $14.720 \mp 2.050$ & $11.051 \mp 3.083$ \\
& MS & $20.542 \mp 1.408$ & $17.474 \mp 2.134$ \\
& HS & $27.701 \mp 1.839$ & $24.435 \mp 1.492$ \\
\hline \hline
\end{tabular}

Considering that the best setting for the $q_{0}$ and tournament size combination is sensitive to the change dynamics, we plan to develop an adaptive mechanism as future work. In this study, we chose a simpler approach. For those cases where tournament selection is to be applied, each time we let the tournament size be determined randomly with equal probability from among the five pre-determined tournament size levels. We performed the $q_{0}$ analysis for AbSts and sAbSts based on this scheme. Table 4 shows the final offline error results for various $q_{0}$ settings for AbSts and sAbSts when the tournament sizes are determined randomly. From the table, we selected $q_{0}=0.1$ for AbSts and $q_{0}=0.9$ for sAbSts. For the rest of the experiments, these settings are used. Comparing the final offline error values for these settings with the average and standard deviation values given in Table 3, we observe that the chosen settings provide either better results than these or the results fall within the intervals defined by these.

Finally, we compare our approaches with those obtained using the heuristic selection methods taken from literature, namely RL, CF and ICF. Table 5 shows the results of these comparisons. The better results are marked in bold in the table. As can be seen, RL and ICF are worse than the others for all cases and these differences are statistically significant, with ICF being the worst of all.

ICF aims to emphasize the intensification component of the generic CF by automatically increasing the weight of relevant components as soon as there is improvement. Diversification, on the other hand, is introduced at a gradually increasing rate. This property works in solving stationary combinatorial optimisation problems as shown in [7], but not in dynamic environments. The changes in the environment mislead ICF and it gets worse than CF in all cases. It was shown in $[9,10]$ that $\mathrm{CF}$ outperforms RL for all tested change dynamics using 
Table 4. Final offline error results of various $q_{0}$ settings for AbSts and sAbSts with random tournament sizes under the tested change frequency-severity pairs

\begin{tabular}{l|c|ccc|ccc|ccc}
\hline \hline \multirow{2}{*}{ Algorithm } & \multirow{2}{*}{$q_{0}$} & \multicolumn{3}{|c|}{ LF } & \multicolumn{3}{c|}{ MF } & \multicolumn{3}{c}{ HF } \\
\cline { 3 - 10 } & & LS & MS & HS & LS & MS & HS & LS & MS & HS \\
\hline \multirow{4}{*}{ AbSts } & 0.0 & 3.844 & 8.516 & 10.901 & 5.236 & 10.231 & 13.033 & 13.434 & 18.360 & 25.459 \\
& 0.1 & 4.063 & 8.019 & 10.940 & 5.069 & 9.566 & 12.853 & 13.486 & 19.035 & 25.256 \\
& 0.3 & 3.744 & 8.287 & 11.107 & 5.172 & 9.974 & 13.192 & 13.120 & 19.325 & 25.327 \\
& 0.5 & 3.932 & 8.086 & 11.877 & 5.012 & 10.201 & 13.360 & 12.843 & 18.345 & 25.949 \\
& 0.7 & 3.916 & 8.322 & 11.777 & 5.150 & 9.689 & 13.176 & 12.872 & 18.833 & 26.593 \\
& 0.9 & 4.030 & 8.778 & 12.011 & 4.924 & 11.963 & 12.958 & 13.861 & 21.461 & 30.139 \\
& 1.0 & 3.987 & 9.977 & 11.735 & 5.460 & 10.791 & 14.618 & 14.640 & 22.261 & 30.589 \\
\hline \multirow{4}{*}{ AbSts } & 0.0 & 4.150 & 8.194 & 10.369 & 5.051 & 10.111 & 12.995 & 12.236 & 17.250 & 24.222 \\
& 0.1 & 4.235 & 8.487 & 10.715 & 5.215 & 10.104 & 13.045 & 12.804 & 18.561 & 24.716 \\
& 0.3 & 3.675 & 8.367 & 11.948 & 4.923 & 10.454 & 12.438 & 11.381 & 17.892 & 24.151 \\
& 0.5 & 3.906 & 8.425 & 11.009 & 4.660 & 9.836 & 13.037 & 10.098 & 16.743 & 24.883 \\
& 0.7 & 4.077 & 8.632 & 11.509 & 4.643 & 10.308 & 13.190 & 9.678 & 16.929 & 23.911 \\
& 0.9 & 4.030 & 8.996 & 11.735 & 4.635 & 10.025 & 12.387 & 9.117 & 16.760 & 23.416 \\
& 1.0 & 4.746 & 10.567 & 12.595 & 7.699 & 12.250 & 14.559 & 13.404 & 18.133 & 24.266 \\
\hline \hline
\end{tabular}

the MPB. Therefore, the poor performance of RL in the current experiments is also to be expected.

The results show that all versions of the proposed heuristic selection scheme provide better results than CF, except for the LF-LS and MF-LS cases, however, the results are close. Among the versions of the proposed heuristic selection scheme, sAbSrw provides the better results in most cases.

Table 5. Final offline error results for the proposed heuristic selection schemes and RL, CF and ICF. Here, for both AbSrw and sAbSrw $q_{0}=0.5$, for AbSts $q_{0}=0.1$ and for $\operatorname{sAbSts} q_{0}=0.9$ with random tournament size settings

\begin{tabular}{l|ccc|ccc|ccc}
\hline \hline \multirow{2}{*}{ Algorithm } & \multicolumn{4}{|c|}{ LF } & \multicolumn{3}{c|}{ MF } & \multicolumn{3}{c}{ HF } \\
\cline { 2 - 10 } & LS & MS & HS & LS & MS & HS & LS & MS & HS \\
\hline AbSrw & 3.720 & 8.114 & $\mathbf{1 0 . 5 8 0}$ & 4.640 & 9.601 & 13.051 & 9.404 & 15.461 & 24.260 \\
sAbSrw & 3.644 & $\mathbf{7 . 4 0 2}$ & 10.990 & 4.180 & $\mathbf{8 . 6 9 8}$ & 12.421 & $\mathbf{7 . 9 3 2}$ & $\mathbf{1 4 . 9 3 5}$ & $\mathbf{2 2 . 9 0 4}$ \\
AbSts & 4.063 & 8.019 & 10.940 & 5.069 & 9.566 & 12.853 & 13.486 & 19.035 & 25.256 \\
sAbSts & 4.030 & 8.996 & 11.735 & 4.635 & 10.025 & $\mathbf{1 2 . 3 8 7}$ & 9.117 & 16.760 & 23.416 \\
\hline CF & $\mathbf{3 . 5 1 8}$ & 9.801 & 11.881 & $\mathbf{4 . 0 9 4}$ & 10.948 & 13.601 & 7.976 & 15.670 & 24.390 \\
ICF & 10.065 & 15.290 & 18.141 & 11.043 & 19.688 & 20.762 & 19.119 & 27.285 & 32.785 \\
RL & 4.427 & 9.046 & 12.179 & 5.968 & 12.440 & 14.540 & 10.285 & 15.685 & 24.569 \\
\hline \hline
\end{tabular}




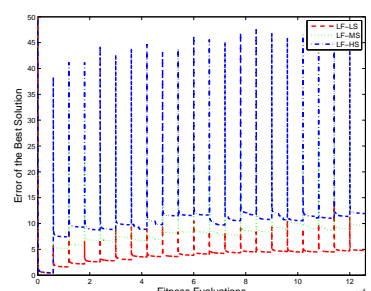

(a)

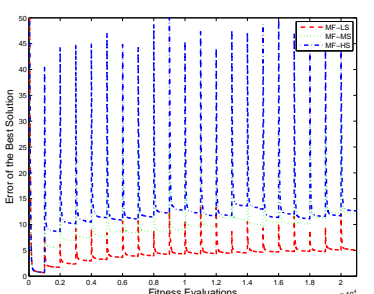

(b)

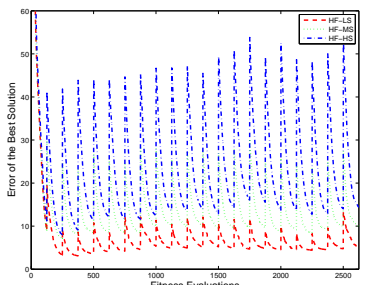

(c)

Fig. 1. Illustration of the tracking ability of sAbSrw for (a) low, (b) medium, and (c) high frequency of change

Due to lack of space, we cannot provide all the statistical comparison tables. Table 6 provides a summary which shows that sAbSrw performs the best with a high level of tracking ability (Figure 4) in the overall based on the number of cases in which this performance difference is either statistically significant or better than the other approaches. sAbSrw is better than CF in many cases, but in several others, their performance is comparable. However, the most important issue is the fact that sAbSrw (and also all the other proposed variants) are more suitable to be used in dynamic environments than RL, CF and ICF because the proposed heuristic selection schemes do not require any special actions to be performed when the environment changes, whereas for the others, right after an environment change, the last solution candidate in the previous environment needs to be re-evaluated. This is a drawback for two reasons: it makes change detection necessary and it also wastes fitness evaluations, especially in environments where the change frequencies are very high.

Table 6. Summary of statistical significance comparisons, where $s+(\geq)$ is the total number of times for which an algorithm was statistically significantly (slightly) better than the others (with no statistical significance), $s-(\leq)$ is the vice versa.

\begin{tabular}{l|c|c|c|c||l|c|c|c|c}
\hline \hline Algorithm & $s+$ & $s-$ & $\geq$ & $\leq$ & Algorithm & $s+$ & $s-$ & $\geq$ & $\leq$ \\
\hline sAbSrw & 15 & 0 & 34 & 5 & CF & 13 & 1 & 18 & 22 \\
AbSrw & 12 & 0 & 24 & 18 & ICF & 0 & 54 & 0 & 0 \\
AbSts & 10 & 9 & 16 & 19 & RL & 11 & 7 & 3 & 33 \\
sAbSts & 10 & 0 & 23 & 21 & & & & & \\
\hline \hline
\end{tabular}

\section{Conclusion}

In this paper, we proposed a new heuristic selection scheme for selection hyperheuristics, especially for use in dynamic environments. In previous studies $[9,10,15]$, 
existing heuristic selection mechanisms were tested in various types of dynamic environments and those that incorporated some form of online learning were shown to be successful. One drawback of these approaches for dynamic environments is that they require the re-evaluation of the last candidate solution in the previous environment for score calculation. As well as wasting computing resources for the re-evaluation, this also means that the algorithm needs to detect when the environment changes. The proposed heuristic selection does not require any special actions when the environment changes. On top of this advantage, the test results also show that the proposed heuristic selection scheme provides slightly better results than the best of the tested heuristic selection schemes found previously to be successful in dynamic environments. The results are very promising and they promote further study. A drawback of the proposed variants of the method is that a couple of parameters is introduced and in some cases, performance is sensitive to their setting. Our future work will focus on enhancing the proposed approach by developing adaptive mechanisms to alleviate the need for parameter tuning and better acceptance schemes for dynamic environment problems.

\section{References}

1. Branke, J.: Memory enhanced evolutionary algorithms for changing optimization problems. In: Congress on Evolutionary Computation CEC 99. vol. 3, pp. 18751882. IEEE (1999)

2. Branke, J.: Evolutionary optimization in dynamic environments. Kluwer (2002)

3. Burke, E.K., Hyde, M., Kendall, G., Ochoa, G., Özcan, E., Rong, Q.: A survey of hyper-heuristics. Tech. rep. (2009)

4. Cowling, P., Kendall, G., Soubeiga, E.: A hyper-heuristic approach to scheduling a sales summit. In: Practice and Theory of Automated Timetabling III : Third International Conference, PATAT 2000. LNCS, vol. 2079. Springer (2000)

5. Cruz, C., Gonzalez, J., Pelta, D.: Optimization in dynamic environments: a survey on problems, methods and measures. Soft Computing - A Fusion of Foundations, Methodologies and Applications 15, 1427-1448 (2011)

6. Dorigo, M., Stützle, T.: Ant Colony Optimizations. MIT Press (2004)

7. Drake, J.H., Özcan, E., Burke, E.K.: An improved choice function heuristic selection for cross domain heuristic search. In: Parallel Problem Solving from Nature PPSN XII. Springer Berlin Heidelberg (2012)

8. Eiben, A.E., Smith, J.E.: Introduction to Evolutionary Computing. Spring (2003)

9. Kiraz, B., Uyar, A.S., Özcan, E.: An investigation of selection hyper-heuristics in dynamic environments. In: Proc. of the int. conf. on Applications of EC - Vol. I. EvoApplications'11, vol. 6624, pp. 314-323 (2011)

10. Kiraz, B., Uyar, A.S., Özcan, E.: Selection hyper-heuristics in dynamic environments. Journal of the Operational Research Society (to appear)

11. Morrison, R.W.: Designing evolutionary algorithms for dynamic environments. Springer (2004)

12. Nareyek, A.: Choosing search heuristics by non-stationary reinforcement learning. In: Metaheuristics: Computer Decision-Making. pp. 523-544. Kluwer Academic Publishers (2001) 
13. Özcan, E., Misir, M., Ochoa, G., Burke, E.K.: A reinforcement learning - greatdeluge hyper-heuristic for examination timetabling. International Journal of Applied Metaheuristic Computing 1(1), 39-59 (2010)

14. Özcan, E., Ş. Uyar, A., Burke, E.: A greedy hyper-heuristic in dynamic environments. In: GECCO 2009 Workshop on Automated Heuristic Design: Crossing the Chasm for Search Methods. pp. 2201-2204 (2009)

15. Uludag, G., Kiraz, B., Etaner-Uyar, A.S., Özcan, E.: A framework to hybridize pbil and a hyper-heuristic for dynamic environments. In: PPSN (2). LNCS, vol. 7492, pp. 358-367. Springer-Verlag, Berlin, Heidelberg (2012)

16. Yang, S., Ong, Y.S., Jin, Y. (eds.): Evolutionary Computation in Dynamic and Uncertain Environments, Studies in Computational Int., vol. 51. Springer (2007) 